\title{
Sistema financeiro, sistema de inovação e desenvolvimento regional: um estudo sobre a relação entre crédito e inovação para os estados da região sudeste brasileira
}

Daniela Almeida Raposo Torres ${ }^{1}$

Filipe Carvalho Vieira ${ }^{2}$

\begin{abstract}
Aline Cristina Cruz ${ }^{3}$
Resumo: Este artigo objetiva identificar teórica e empiricamente a relação entre um eficiente sistema financeiro e um sistema de inovação consolidado para os estados do Sudeste brasileiro entre 2000 e 2012, com base na literatura Keynesiana e Schumpeteriana. Com base no método de dados em painel, identifica-se que tanto o montante de depósitos totais do sistema financeiro, quanto o volume estadual de crédito possuem relação positiva com o dinamismo econômico. Já a relação entre preferência pela liquidez e crédito mostrou-se inversamente proporcional, conforme prediz a teoria. Ademais, confirma-se a importância do learning-by-doing, da renda interna bruta estadual e do crédito financeiro para aumentos no índice de progresso tecnológico das economias estaduais do Sudeste brasileiro. Em suma, conclui-se pela necessidade de políticas de estímulo ao desenvolvimento do sistema financeiro e do sistema regional de inovações, visando ao crescimento econômico sustentável.
\end{abstract}

Palavras-chave: Inovação; Crédito; Desenvolvimento Regional.

JEL: G21, L16, R11.

1 Professora Adjunta III, Departamento de Ciências Econômicas, Universidade Federal de São João del Rei (UFSJ).

2 Bacharel em Ciências Econômicas. E-mail: filipecarvalho.vieira@gmail.com

3 Professora Adjunta III, Departamento de Ciências Econômicas, Universidade Federal de São João del Rei (UFSJ). 


\title{
Financial system, innovation system and regional development: a study on the relationship between credit and innovation to the states of the southeast region of Brazil
}

\begin{abstract}
This paper aimed to identify theoretically and empirically the relationship between an efficient financial system and a consolidated innovation to the states of the Brazilian Southeast region between 2000 and 2012, based on Keynesian literature and the theoretical framework of Schumpeter. In the analysis of panel data, it is evident that the amounts of total deposits in the financial system and the state credit volume have positive relationship with economic dynamism. However, the relationship between credit and liquidity preference was inversely proportional, as the theory predicts. The second panel estimated confirms the importance of learning-by-doing, the state income and financial credit to increases in the rate of technological progress of the economies of Southeast Brazil. In short, it is concluded by the need to adopt policies to encourage the development of both the financial system, as the regional innovation system of the economy, aiming to raise its growth rate sustainably.
\end{abstract}

Keywords: Innovation; credit; Regional Development.

JEL: G21, L16, R11.

\section{Introdução}

Há uma vasta literatura sobre a importância do progresso tecnológico para o fomento do crescimento e desenvolvimento econômico. De acordo com a corrente de pensamento Evolucionária ${ }^{1}$, o progresso tecnológico depende do Sistema de Inovação (SI) de um país e/ou região. Segundo Sbicca e Pelaez (2006), os SI dizem respeito ao conjunto de instituições públicas e privadas que contribuem nos âmbitos macro e microeconômico para o desenvolvimento, execução, apoio, fomento e difusão de novas tecnologias. A respeito disso, Archibugi e Michie (1997) demonstram que os SI consolidados necessitam de substanciais componentes inter-relacionados de maneira eficiente, quais sejam: centros de pesquisas, universidades, firmas e setor financeiro, de forma

1 Ver em Nelson e Winter (1982) sobre o desenvolvimento dos antecedentes e das premissas básicas da abordagem evolucionária. 
que o processo de inovação impacte positiva e diretamente no desenvolvimento econômico e social. Torres (2009) destaca a natureza destas interações de dependência do arcabouço institucional do país, de suas especificidades culturais, históricas e do sistema educacional. Portanto, cada país, de acordo com suas peculiaridades, traça ao seu tempo o desenvolvimento do seu SI.

Ademais, é consenso na literatura que a inovação tecnológica é essencial para manutenção do crescimento. Em termos econômicos, a tecnologia deve ser encarada como o conhecimento humano aplicado para ampliar a produção. Portanto, a nação ou região deve possuir mão de obra qualificada para proporcionar ambiente apropriado à criação e manutenção da capacitação tecnológica (Oliveira, 2001). Além disso, a capacidade do país de modificar seu sistema econômico depende fundamentalmente da organização de sistemas de inovação exitosos.

Entretanto, Chesnais e Sauviat (2005, p. 161) alerta, que muito pouca atenção tem sido dispensada "às condições sob as quais os principais participantes dos sistemas de inovação - firmas, governo e órgãos públicos - comandarão os financiamentos necessários para a realização de investimentos em inovação de longo prazo". A literatura têm dado ênfase às instituições, à rede e aos processos interativos de aprendizado, por sua vez, dependentes de fontes de financiamento para Pesquisa e Desenvolvimento (P\&D) e investimento de longo prazo em equipamentos, infraestrutura e treinamento de mão de obra.

Sobre o assunto, Schumpeter (1982) discute a importância do crédito para o processo de inovação, pois os empreendedores são forças motrizes no processo de inovação e devem ter êxito ao convencer os bancos a fornecerem o crédito para inovação. Isso revela a dependência de recursos financeiros gerados pela expansão do crédito via sistema financeiro.

Independentemente de ser pública ou privada a fonte dos recursos, os mecanismos destinados a financiar investimentos em inovação tecnológica, como mencionado por Corder e Salles-Filho (2005), são diferentes daqueles voltados ao financiamento convencional. Isso por conta dos riscos envolvidos e do tempo de retorno, os quais tendem a serem superiores àqueles normalmente vinculados aos demais investimentos. Por isso motivo, e devido às instabilidades dos resultados e de sua apropriação, investir em inovações exige um novo conceito de financiamento a partir da abordagem pós-keynesiana.

Diante disso, o objetivo deste estudo é identificar a relação existente entre o sistema financeiro e o sistema de inovação, para os estados da região Sudeste entre os anos de 2000 e 2012, a partir da abordagem neoschumpeteriana e da perspectiva pós-keynesiana. A justificativa para este período é a possibilidade de uma base de dados mais completa, principalmente quanto às patentes estaduais, cujo início das informações é o ano 2000. Para tal, a estrutura do trabalho contempla, além da introdução e conclusões, a seção um que expõe os fundamentos teóricos de Keynes e os neoschumpeterianos, seguida da 
seção dois de referencial analítico. Em seguida, são descritos o método de pesquisa e os resultados obtidos.

\section{Referencial Teórico}

O presente trabalho respalda-se na abordagem teórico-histórica de Keynes e Schumpeter já consolidadas na literatura econômica. Além disso, de maneira complementar, são utilizadas as teorias pós-keynesiana e neoschumpeteriana sobre financiamento, investimento e inovação. O tratamento keynesiano e pós-keynesiano da moeda confere a esta papel não neutro em relação às variáveis reais da economia. Segundo Keynes (1973, p.408-9),

... a moeda joga um papel próprio e afeta motivações e decisões, sendo, em suma, um dos fatores operativos na situação, de forma que o curso dos eventos não pode ser predito, tanto no longo quanto no curto período, sem o conhecimento do comportamento da moeda entre o primeiro e o último estados.

Ademais, a alocação de parcela da riqueza do indivíduo sob forma de moeda é racional à luz da incerteza ${ }^{2}$. A preferência pela liquidez "é representada por uma escala do volume dos recursos do indivíduo, medidos em termos monetários ou em unidades de salários, que este deseja conservar em forma de moeda em diferentes circunstâncias" (Keynes, 1983, cap. 13). Assim, quanto maior a incerteza percebida pelos agentes, maior seu desejo de reter moeda para aplacar sua insegurança quanto ao futuro, o qual depende das decisões e comportamento de todos os agentes que operam nessa economia. De forma que, quando as expectativas dos agentes são pessimistas, estes podem demandar algo que lhes dê segurança e flexibilidade hoje para enfrentar o amanhã. Um ativo de elevada liquidez seria o refúgio dado à imprevisibilidade, além de conferir flexibilidade para que se possam aproveitar as melhores oportunidades de lucro. Logo, coeteris paribus, quanto mais incerto é o futuro, maior é a preferência pela liquidez dos agentes, e consequentemente maior a demanda por moeda, ativo de maior liquidez na economia (Carvalho, 2000).

No que diz respeito à taxa de juros, segundo Keynes, esta é a recompensa do indivíduo por não entesourar, isto é, o prêmio por abrir mão da liquidez (Carvalho, 2000; Paula, 2003). Segundo Keynes (1999, p.174),: 
"A quantidade de moeda e o montante da mesma requerida pela circulação ativa para as transações correntes (que dependem principalmente do nível de renda nominal) determinam o montante disponível para saldos inativos, isto é, para o entesouramento. A taxa de juros é o fator que ajusta na margem a procura de dinheiro para entesouramento à oferta de numerário para o mesmo fim".

Portanto, a taxa de juros é determinada pela demanda e oferta de moeda, ativo de liquidez máxima. Para que seu possuidor abra mão das vantagens inerentes a sua propriedade, é exigido um prêmio. Logo, os juros são simultaneamento o preço pago por alguns agentes desejosos de liquidez e o prêmio embolsado pelos emprestadores. Quanto maior a incerteza quanto ao futuro, coeteris paribus, maior a preferência pela liquidez, maior, portanto, a taxa de juros, cujos efeitos são contracionistas sobre o produto e o emprego.

Nessa linha, cabe mencionar que Keynes (1930) no Tratado Sobre Moeda identifica dois circuitos de circulação monetária: o industrial e o financeiro. $\mathrm{O}$ autor destaca que, no primeiro, a moeda é meio de troca, dando suporte ao giro de bens e serviços na economia. Já com a circulação financeira, a moeda faz girar ativos, podendo ser a própria moeda retida como ativo. Neste caso, a demanda por moeda se daria pelos motivos especulação e precaução. Assim, ao se tornar um ativo, a moeda passa a concorrer com outros pela demanda dos agentes. É importante destacar que, quanto maior o vazamento da circulação industrial para a financeira, mais a atividade econômica tende a decrescer.

Em trabalhos posteriores, Keynes (1988a e 1988b) apresenta o quarto motivo para a demanda por moeda: o motivo finance, no qual o investimento depende da poupança e do finance. Tal motivo para reter liquidez define-se pela liquidez que o empresário precisa reter entre o momento de planejamento de gastos de investimento e o momento no qual o mesmo efetivamente é realizado. A liberação ou a criação de finance seria um pré-requisito do investimento, o que demonstra a relevância do sistema financeiro no processo econômico (Cintra, 1999).

Importante apontar que o investimento depende das expectativas dos capitalistas quanto aos ganhos futuros. Num mundo de incerteza, a escolha dos empresários de investir em ativos ilíquidos associa-se ao elevado grau de confiança no futuro e vice-versa. Dessa forma, a decisão de investir baseia-se em elementos subjetivos, como o animal spirits dos empresários e da eficiência marginal do capital. ${ }^{3}$ Quando há expansão do investimento planejado e da demanda por liquidez não acompanhada pelo adicional na oferta de finance, ocorre elevação da taxa de juros e inibição do investimento. $\mathrm{O}$ aumento da atividade corrente envolve maior demanda de recursos líquidos, a qual não 
pode ser atendida sem elevação da taxa de juros, a menos que os bancos ou o resto do público disponha-se a liberar mais capital à taxa de juros dada. Sobre o investimento realizado, destaca-se que esse eleva a capacidade produtiva da economia, reduzindo a eficiência marginal do capital. Portanto, quando há expansão do investimento planejado e da demanda por liquidez, sem a ampliação da oferta de finance, ocorre elevação da taxa de juros, o que dificulta o investimento.

Para realização dos investimentos, menciona-se que em grande parte os bancos, detentores do crédito, levam em conta a possibilidade de retorno via empréstimos. Com isso, percebe-se que, se as expectativas são menos conservadoras, eleva-se o otimismo e diminui-se a importância do prêmio de liquidez do ativo. Isso ocorre simultaneamente à ampliação da importância da rentabilidade na composição do balanço bancário, passando os bancos a adotarem a postura financeira especulativa. Por exemplo, em momentos de boom econômico, há diminuição substancial da preferência pela liquidez dos bancos e aumento da busca pela rentabilidade, com conseqüente flexibilização dos critérios bancários de concessão de liquidez.

Em economias abertas, os efeitos, negativos e positivos do lado financeiro sobre o lado real são intensificados. Isso porque de acordo com Dow (1993), o sistema financeiro internacional não é neutro, e a distribuição de crédito externo não é uniforme entre os países. Assim, o sistema financeiro afeta o desenvolvimento e o crescimento das economias de modo desigual, podendo favorecer o aumento das divergências de crescimento entre estas (DOW, 1986, 1987 e 1993). Em períodos com tais características, a oferta de finance decresce, de forma que o impacto é negativo na economia nacional, já que esta é totalmente integrada ao sistema financeiro internacional, tal como as economias globalizadas no mundo contemporâneo. Isto equivale a dizer que a abertura financeira amplifica os movimentos da oferta de moeda, pois o sistema financeiro é de fundamental importância para concretização do investimento em inovação. Neste ponto, vale ressaltar que, segundo Romero e Jayme Jr. (2008), a literatura sobre economia regional dá escasso destaque à moeda, sendo os fluxos monetários considerados resultado das diferenças de desenvolvimento entre regiões, e não sua causa.

Desta forma, no que diz respeito às economias em desenvolvimento como o Brasil, estas são classificadas pelo sistema financeiro internacional como especulativas/ponzi, pois apresentam menores graus de desenvolvimento de seu Sistema Nacional de Inovação (Nelson, 1993). Em períodos de ascensão da liquidez mundial em economias centrais e periféricas, estas nações são abastecidas pelo crédito externo. Já na recessão econômica, como mencionado por Torres (2009), o racionamento de crédito é mais intenso em nações com maiores dificuldades de honrar seus compromissos financeiros internacionais, tal como identificado em economias em desenvolvimento. Ademais, em períodos de depressão econômica, ocorre racionamento de crédito disponível 
para investimentos tanto no âmbito do sistema financeiro doméstico, como no internacional. Desta forma, para que a economia continue a prosperar, o sistema financeiro deve incentivar o processo de inovação na indústria e em outros setores da economia (Schumpeter, 1982).

Resende (2005) demonstra que, nas economias de SI imaturo, a vulnerabilidade externa é caráter permanente e estrutural. Ou seja, países com SI deficientes apresentam grande dependência do capital externo, ficando à mercê da liquidez internacional. ${ }^{4}$ Para Perez (2004), nas economias em desenvolvimento, em face à restrição de financiamento interno, o capital financeiro externo apoia o produtivo nos investimentos associados às novas tecnologias. Assim, as condições de desenvolvimento destes países quanto à capacidade de aproveitar as janelas de oportunidades abertas fica comprometida. Neste ponto, Ernst (2000) alerta para a frágil estrutura produtiva destas nações, pois é integrada a empresas sediadas em outros países. Sobre isso, Silva (2011) lembra que economias de elevado grau de restrição externa tornam-se dependentes da poupança externa para financiamento da produção nacional.

Neste contexto, Studart (1995) considera que economias sem mercados financeiros organizados tendem a apresentar grandes dificuldades de desenvolvimento econômico. Isto se dá pelo aumento da preferência pela liquidez, o qual diminui os empréstimos de longo prazo por parte do sistema financeiro. Com a diminuição da taxa de investimento, o crescimento econômico também tende a se reduzir, dificultando os investimentos em inovação, uma vez que a incerteza prevalece. Em suma, esquemas financeiros alternativos como a atuação do Governo tornam-se essenciais para inibir a fragilidade financeira e permitir a sustentação do crescimento econômico.

Feitas as análises sobre o setor bancário e sua capacidade de gerar crédito, faz-se necessária uma breve explanação da abordagem de Schumpeter sobre a relação entre sistema financeiro e o processo de inovação. Segundo Schumpeter (1982), para que a inovação aconteça, o setor bancário deve estar consolidado e em sintonia com os empresários existentes. A literatura schumpeteriana demonstra que a inovação e o progresso técnico são processos endógenos à dinâmica econômica, portanto, a inovação é o fenômeno central da dinâmi

ca capitalista. E, de acordo com a corrente de pensamento Evolucionária, o progresso tecnológico depende do sistema nacional de inovação de um país. ${ }^{5}$

Ademais, Schumpeter (1982) demonstra que o crédito é um dos elementos básicos do processo de desenvolvimento econômico e complemento monetário

4 Neste ponto, destaca-se Perez (2004 e 2009) como importante referência neoschumpetriana da relação existente entre difusão tecnológica e capital financeiro em âmbito internacional. Para mais detalhes, ver Perez, C. (2004). Revoluciones tecnológicas y capital financiero: la dinâmica de las grandes burbujas financieras y las épocas de bonanza. México: Siglo XXI. Perez, C. (2009). Technological revolutions and techno-economic paradigms. Working Papers in Technology Governance and Economic Dynamics, $\mathrm{n}^{\circ} 20$. Technology governance.

$5 \mathrm{O}$ conceito de sistema nacional de inovação (SI) foi elaborado por Freeman, Nelson e Lundvall, numa obra coletiva de sistematização da abordagem evolucionária - "Techinal Change and Economic Theory", organizada por Dosi et al. (1988). 
da inovação. Já Paula (2011) destaca que o crédito tornou-se fator necessário para o desenvolvimento, quando as inovações são feitas por novos empresários sem seus próprios meios de produção. O resultado é que, por meio da criação de moeda bancária, os bancos concedem crédito aos chamados empresários inovadores. Segundo o autor, pode-se observar certa semelhança entre as análises feitas por Schumpeter, com o processo de desenvolvimento sendo alavancado por inovações, e os estudos de Keynes, diferenciando economias com e sem aumento de investimento. Entretanto, Paula (2011) destaca que há diferenças da visão do papel da moeda na economia entre ambos os autores. Especificamente, a moeda na economia monetária de Keynes não tem o mesmo sentido do crédito na visão de Schumpeter. Para Keynes, a moeda corresponde ao finance, com o sentido de reserva de valor, em função da preferência pela liquidez, e, portanto, trata-se de demanda preventiva ou especulativa e não transacional. De todo modo, Keynes e Schumpeter concordam que a moeda-crédito criada pelos bancos desempenha papel importante no financiamento da atividade econômica, e em especial, no investimento produtivo ou em inovações, o que revela a complementaridade entre as teorias destes teóricos. Em suma, demonstra-se aqui a importância do papel moeda para um sistema financeiro eficaz, o qual tende a contribuir para o desenvolvimento econômico. Deste modo, diante dos objetivos do estudo, a seguir apresenta-se a metodologia.

\section{Metodologia}

\section{1- Construção de indicadores}

Nesta seção aborda-se o método de pesquisa para estabelecer as relações existentes entre o grau de desenvolvimento do SI e do sistema financeiro para os estados do Sudeste do Brasil. Especificamente, para representar a dimensão monetário-financeira, tem-se as variáveis: crédito bancário; depósitos à vista e a prazo e número de agências de cada unidade federativa.

Tais dados permitem algumas inferências sobre o comportamento dos bancos, especialmente, quanto à decisão de fornecer crédito em função da base de depósitos regional. A proposta é o cálculo para cada unidade federativa de um índice de comportamento dos bancos quanto à forma de oferecimento de liquidez ao sistema produtivo. Seguindo a metodologia de Crocco (2005), estima-se a preferência pela liquidez dos bancos (PLB) desta forma:

$$
P L B=\frac{\text { Depósitos à vista }}{\text { Crédito }}
$$


em que depósitos a vista representam os recursos mais líquidos dos bancos, enquanto os dados sobre crédito representam sua alocação de recursos de menor liquidez, ou seja, sua disposição em liberar empréstimos. Desta forma, quanto menor a disposição do banco em emprestar, maior sua preferência pela liquidez. $\mathrm{O}$ que se espera é que, em regiões com maior incerteza econômica, o nível de empréstimos seja menor, devido à maior preferência pela liquidez dos bancos frente a essa incerteza. Deste modo, a expectativa é de que o nível de desenvolvimento e geração de inovação seja baixo em razão do ambiente de maior incerteza e do menor otimismo dos investidores.

Considerando-se que o progresso tecnológico pode ser medido pelo grau de desenvolvimento (relativo) do SI, usa-se como medida de progresso tecnológico a variável TEC, a exemplo de Herskovic, Ribeiro e Albuquerque (2008). Trata-se de uma proxy para o progresso científico-tecnológico do estado $i$, como descrito abaixo:

$$
\mathrm{TEC}_{\mathrm{it}}=1 / 2\left(\ln \mathrm{ART}_{\mathrm{it}}+\ln \mathrm{PAT}_{\mathrm{it}}\right)
$$

na qual lnArt ${ }_{\text {it }}$ é o logaritmo natural da média do número de artigos publicados por milhão de habitantes e lnPat ${ }_{i t}$ é o logaritmo natural da média do número de pedidos de patentes por milhão de habitantes, para o estado $i$. Segundo o estudo, sendo um índice sintético, esta medida é sujeita a problemas, pois ao transformar o progresso científico-tecnológico em um número, pode-se ignorar vários aspectos qualitativos, à medida que cada país tem um desenvolvimento histórico distinto do sistema nacional de inovação. No entanto, a proposta é de mérito, pois permite analisar a relação não corriqueira entre dimensão inovativa e financeira.

\subsection{Modelo Econométrico}

Para testar a hipótese de que o grau de desenvolvimento do sistema financeiro dos estados afeta o grau de desenvolvimento dos seus sistemas de inovação, primeiramente são testados os determinantes da oferta de crédito por estado. Especificamente, testa-se a relação entre o volume de crédito e preferência pela liquidez dos bancos (PLB), PIB estadual (PIB) e depósitos totais (DEP) ${ }^{6}$, como mostra a equação (3):

$$
\text { Créd }_{i t}=\beta_{1}+\beta_{2} P L B_{t}+\beta_{3} P I B_{t}+\beta_{4} D E P_{t}+\varepsilon_{i}
$$


O PIB estadual é incluído no modelo para representar o nível de atividade econômica, assumindo que, quanto maior seu volume, maior a demanda e a oferta de crédito dos bancos. Já os depósitos representam a totalidade de recursos disponíveis dos bancos intra-estado, supostamente apresentando também relação positiva com o nível de crédito. A proposta é identificar se a preferência pela liquidez dos bancos influencia o montante total de crédito disponibilizado nestes estados.

A segunda regressão considera a relação entre variáveis financeiras e tecnológicas e o nível tecnológico de cada estado, de acordo com a equação (4):

$$
T E C_{i t}=\beta_{1}+\beta_{2} \text { Créd }_{i}+\beta_{3} P A T_{i-1}+\beta_{4} P I B_{i}+\varepsilon_{i}
$$

em que $T E C_{t}$ é a variável dependente proxy do progresso científico-tecnológico estadual. Já as variáveis explicativas são: volume de crédito (Créd $\left.{ }_{i t}\right)$; número de patentes e desenhos industriais depositados no INPI defasado $\left(\mathrm{PAT}_{\mathrm{it}-1}\right)$ e o PIB estadual -PIB ${ }_{\text {itt }}$. Esta equação procura avaliar se o crédito, com suposta relação inversa com a preferência pela liquidez, tem impacto no desenvolvimento tecnológico de cada estado (TEC).

A variável crédito representa o acesso da população a recursos financeiros necessários ao fomento de atividades produtivas, entre estas as inovativas. A relação esperada é que, quanto menor a preferência pela liquidez, maior o prazo do crédito e maior o financiamento da atividade inovativa. Isto equivale a dizer que a queda da preferência pela liquidez tem impacto sobre o desenvolvimento regional. Por outro lado, o crédito disponibilizado colabora também para liberar recursos para as atividades de P\&D, motivando assim a inovação. Além disso, espera-se que o otimismo dos agentes, no contexto de baixa preferência pela liquidez, incentive a atividade inovativa e a concessão de crédito. Por fim, o número de patentes dos períodos anteriores busca representar o componente de learning-by-doing da inovação e fatores relacionados aos benefícios das inovações passadas. Em muitos casos, uma inovação encaminha para novas descobertas, o que possibilita o registro de novas patentes resultantes da inovação inicial.

Considerando-se a base de dados utilizada, usou-se dos modelos de dados em painel. Sobre tal aplicação, Gujarati (2006) enumera que o método considera explicitamente a heterogeneidade entre as unidades estudadas; proporciona dados mais informativos com mais graus de liberdade e maior eficiência; e minimiza o viés decorrente da agregação de unidades em grandes conjuntos.

Dito isso, duas abordagens são testadas: o modelo de efeito fixo e o modelo de efeito aleatório. A proposta é separar os fatores não observados que afetam a variável dependente em dois tipos: os que são constantes (efeito fixo) e os que variam ao longo do tempo (efeito aleatório). O primeiro caso leva em con- 
sideração os efeitos específicos atrelados às unidades individuais, no caso os estados, principalmente, quando existe grande heterogeneidade na amostra.

A diferença entre o modelo de efeitos aleatórios e o de efeitos fixos é a não correlação entre o efeito não observado e as variáveis explicativas. Para tal, aplica-se o teste de Breusch-Pagan (Greene, 2002), que identifica se o modelo de efeito aleatório é melhor que o modelo pooling. Já o teste de especificação de Hausman (1978) segue a hipótese nula de inexistência da correlação supracitada, seguindo a estatística de teste Qui- Quadrado. No caso de haver correlação, a opta-se pelo estimador de efeito fixo.

Para atestar a inexistência de autocorrelação serial dos erros, segue-se Wooldridge (2010) que segue a distribuição F. Aplica-se também o teste baseado na distribuição $\chi^{2}$ (Quadrado) proposto por Greene (2002), para testar se os grupos apresentam variâncias idênticas (group wise heterokesdasticity test).

No que concerne aos dados, as informações sobre crédito bancário disponibilizado em cada estado da região Sudeste e depósitos à vista e a prazo foram obtidas junto à base de dados do Banco Central. Já os dados de patentes depositadas foram coletados junto ao INPI (Instituto Nacional de Propriedade Industrial, 2013) e os dados de artigos publicados mediante consulta ao ISI (Web of. Knowledge - IP \& Science, 2013). ${ }^{7}$ Para os dados estaduais de população, consultou-se o IPEADATA (2013), assim como para identificar o nível de atividade econômica de cada estado brasileiro (PIB estadual). Ademais, os dados são anuais e deflacionados em relação ao IGP-DI, considerando como período base $o$ ano de 2000, e no modelo econométrico, foram logaritmizadas.

\section{Resultados}

Primeiramente, usa-se da Estatística Descritiva das variáveis propostas para avaliar a relação entre preferência pela liquidez e capacidade inovativa a partir da Tabela 1. É possível observar, a princípio, que os índices de PLB da região Sudeste enquadram-se na teoria de Keynes (1983, cap.13) sobre as atividades bancárias. Percebe-se ainda que o Espírito Santo é o estado com maior preferência pela liquidez, seguido de Rio de Janeiro, Minas Gerais e São Paulo. Isso possibilita dizer, portanto, que maior pode ser a incerteza percebida pelos agentes quanto ao futuro da região capixaba, ou ainda que os agentes deste estado parecem apresentar maior grau de especulação/ponzi. Isso dá indícios de que estados menos desenvolvidos tecnologicamente tendem a apresentar indicadores de PLB superiores aos dos estados mais desenvolvidos.

7 A variável de patentes foi construída a partir de informações disponibilizadas em http://www.mct. gov.br/index.php/content/view/342297/Brasil__Pedidos_de_patentes_depositados_sup_1_sup__ no_Instituto_Nacional_da_Propriedade_Industrial_INPI_por_residentes_segundo_tipos_por_ unidade_da_federacao.html, acesso em novembro de 2013. A variável de artigos foi construída a partir de dados disponibilizados em http://apps.webofknowledge.com/WOS_AdvancedSearch_input. do?product=WOS\&SID=3Ba9lklv28gfk4kQQLb\&search_mode=AdvancedSearch. Acesso em abril de 2014. 
TORRES, D. A. R; VIEIRA, F. C.,; CRUZ, A. C. Sistema Financeiro, sistema de inovação e desenvolvimento....

TABELA 1 - ÍNDICE DE PREFERÊNCIA PELA LIQUIDEZ DOS BANCOS, ESTADOS DA REGIÃO SUDESTE, 2000 A 2012

\begin{tabular}{c|c|c|c|c|c}
\hline $\begin{array}{c}\text { Período/ } \\
\text { Região }\end{array}$ & $\begin{array}{c}\text { Minas } \\
\text { Gerais }\end{array}$ & $\begin{array}{c}\text { Espírito } \\
\text { Santo }\end{array}$ & $\begin{array}{c}\text { Rio de } \\
\text { Janeiro }\end{array}$ & $\begin{array}{c}\text { São } \\
\text { Paulo }\end{array}$ & Sudeste \\
\hline 2000 & 0,172 & 0,202 & 0,133 & 0,090 & 0,149 \\
2001 & 0,197 & 0,221 & 0,187 & 0,086 & 0,173 \\
2002 & 0,223 & 0,291 & 0,224 & 0,095 & 0,208 \\
2003 & 0,213 & 0,329 & 0,229 & 0,098 & 0,217 \\
2004 & 0,212 & 0,328 & 0,266 & 0,109 & 0,229 \\
2005 & 0,192 & 0,319 & 0,277 & 0,114 & 0,225 \\
2006 & 0,225 & 0,295 & 0,297 & 0,109 & 0,231 \\
2007 & 0,214 & 0,288 & 0,298 & 0,101 & 0,225 \\
2008 & 0,166 & 0,292 & 0,222 & 0,084 & 0,191 \\
2009 & 0,156 & 0,239 & 0,206 & 0,083 & 0,171 \\
2010 & 0,147 & 0,232 & 0,191 & 0,089 & 0,165 \\
2011 & 0,125 & 0,204 & 0,177 & 0,073 & 0,145 \\
2012 & 0,100 & 0,178 & 0,161 & 0,061 & 0,125 \\
\hline
\end{tabular}

Fonte: Elaboração própria com dados do BACEN (2013).

A seguir, a Tabela 2 apresenta os números relativos a patentes, artigos e PIB, bem como o número absoluto em bilhões de reais de crédito. Tais informações evidenciam que os estados com maiores participações na renda nacional apresentam quantidades significativas de publicações científicas, patentes e crédito. Percebe-se também que os dados correspondem à proposta teórica descrita anteriormente de atestar a influência do sistema financeiro sobre a produção de inovação. Ademais, na comparação entre os dados das Tabelas 1 e 2, a saber: PLB, porcentagem de patentes e artigos, acredita-se que, quanto menor a preferência pela liquidez, maior a disposição dos agentes em investir em ativos menos líquidos, podendo motivar o investimento em atividades inovativas, com risco mais elevados, mas também maior retorno. Além disso, em ambientes de incerteza menor, o investimento em ativos inovativos torna-se mais presente na economia, o que contribui para o amadurecimento do sistema de inovação. 
TORRES, D. A. R; VIEIRA, F. C.,; CRUZ, A. C. Sistema Financeiro, sistema de inovação e desenvolvimento....

TABELA 2 - PERCENTUAL MÉDIO DE PATENTES, ARTIGOS E PIB ESTADUAL E CRÉDITO BANCÁRIO, ESTADOS DA REGIÃO SUDESTE, 2000 A 2012

\begin{tabular}{|c|c|c|c|c|}
\hline Variáveis & $\begin{array}{l}\text { Espírito } \\
\text { Santo }\end{array}$ & $\begin{array}{l}\text { Minas } \\
\text { Gerais }\end{array}$ & $\begin{array}{l}\text { Rio de } \\
\text { Janeiro }\end{array}$ & São Paulo \\
\hline $\begin{array}{l}\text { Percentual de } \\
\text { patentes estadual/ } \\
\text { patentes Brasil }\end{array}$ & 1,44 & 8,68 & 7,97 & 44,25 \\
\hline $\begin{array}{l}\text { Percentual de } \\
\text { artigos estadual/ } \\
\text { artigos Brasil }\end{array}$ & 0,35 & 5,48 & 9,45 & 26,26 \\
\hline $\begin{array}{l}\text { Percentual PIB } \\
\text { estadual/PIB } \\
\text { Brasil }\end{array}$ & 2,13 & 9,01 & 11,38 & 33,87 \\
\hline $\begin{array}{l}\text { Crédito em bilhões } \\
\text { de reais }\end{array}$ & 43,53 & 286,49 & 373,56 & 2851,04 \\
\hline
\end{tabular}

Fonte: Elaboração própria com dados da pesquisa.

Os resultados econométricos obtidos consideram dois modelos de dados em painel, de forma a acompanhar cada estado do Sudeste brasileiro de 2000 a 2012. Conforme já discutido, há a possibilidade de estimação de um modelo de efeitos fixos ou de efeitos aleatórios. Na aplicação do teste de Hausman, conclui-se pela rejeição da hipótese nula de que o modelo de efeitos aleatórios é o mais indicado para ambos os modelos estimados.

No que se refere aos pressupostos básicos, a primeira estimação (Tabela 3) apontou presença de autocorrelação entre os erros, apesar da ausência de heterocedasticidade. Para eliminar tal violação, o modelo foi estimado novamente, usando o método de erros robustos, de forma que os estimadores de efeitos fixos tornam-se eficientes, mediante erros não serialmente correlacionados e também homocedásticos. Já o modelo proposto para avaliar as variáveis determinantes do nível tecnológico (Tabela 4) apresentou variância não constante dos erros e autocorrelação entre os mesmos. Sob tal condição, também se procedeu à outra estimação, mantendo o modelo de efeitos fixos, mas a partir do método de erros robustos.

Na Tabela 3, expõem-se os resultados das relações obtidas entre as variáveis explicativas (depósitos totais, renda interna bruta e preferência pela liquidez) e o volume de crédito estadual. Em primeiro lugar, observa-se o coeficiente negativo e estatisticamente significativo para a relação entre crédito estadual e a proxy de preferência pela liquidez (PLB). Tal resultado permite inferir que o cenário de aumento da preferência pela liquidez nestes estados está associado ao decréscimo do valor esperado do volume de crédito para o período.

De acordo com os argumentos teóricos, os bancos, financiadores dos projetos 
TORRES, D. A. R; VIEIRA, F. C.,; CRUZ, A. C. Sistema Financeiro, sistema de inovação e desenvolvimento....

de investimento, têm preferência pela liquidez em meio a ambientes de incerteza. Isso equivale a dizer que a escala de preferência pela liquidez deste tipo de instituição financeira expressa a precaução da firma, cuja atividade envolve resultados incertos quanto ao retorno de suas aplicações. Deste modo, sua estratégia segue o trade off entre rentabilidade e liquidez. Se o banco prefere liquidez à rentabilidade, escolhe ativos mais líquidos em seu portfólio de aplicações. Em cenário oposto, busca ativos menos líquidos ou de maior retorno/risco. Assim, os bancos com preferência pela liquidez podem não acomodar passivamente a demanda por crédito, conforme aponta o primeiro modelo.

TABELA 3 -MODELO DE DADOS EM PAINEL PARA EXPLICAR AS VARIAÇÕES DO VOLUME DE CRÉDITO, ESTADOS DA REGIÃO SUDESTE, 2000-2012

\begin{tabular}{l|c}
\hline \multicolumn{1}{c|}{ Variáveis Explicativas } & Crédito \\
\hline Constante & $3,8110^{\mathrm{ns}}$ \\
& $(0,07010)$ \\
Preferência pela liquidez & $-0,7286^{* * *}$ \\
& $(0,07010)$ \\
Produto Interno Bruto & $0,9600^{* *}$ \\
& $(0,1928)$ \\
Depósitos Totais & $0,1257^{*}$ \\
Coeficiente de determinação intraestados $\left(\mathrm{R}^{2}{ }_{\text {within }}{ }^{*}\right.$ & $(0,0524)$ \\
Coeficiente de determinação entre estados $\left(\mathrm{R}^{2}{ }_{\text {between }}\right)$ & 0,9616 \\
Coeficiente de determinação geral $\left(\mathrm{R}^{2}{ }_{\text {overall }}\right)$ & 0,9986 \\
Erro padrão de $\alpha_{i}$ & 0,9964 \\
Erro padrão de $\varepsilon_{\dot{i s}}$ & 0,1258 \\
Correlação entre os resíduos - Corr $\left(\varepsilon_{\dot{\text { is }}}, \varepsilon_{i}\right)$ & 0,0752 \\
Teste F de Significância Global & 0,7370 \\
\hline
\end{tabular}

Fonte: Resultados da pesquisa.

Nota: Forma Funcional duplo - log. Valores entre parênteses: erro-padrão.

- ns: não significativo, ${ }^{* * *}$ : significativo a $1 \%,{ }^{* *}$ : significativo a $5 \% \mathrm{e}^{*}$ : significativo a $10 \%$.

Com relação à relação entre depósitos totais e montante de crédito, identifica-se relação positiva e estatisticamente significativa, corroborando as hipó- 
teses aqui apresentadas. Sendo os depósitos totais a soma de depósitos à vista e a prazo, o aumento dos depósitos a prazo significa que mais recursos do passivo estão em posições menos líquidas. Assim, há maior margem de manobra dos bancos para alongar o prazo dos recursos do seu ativo, ou seja, podem tornar-se menos líquidos e aumentar a disponibilidade de crédito. Já a conta depósitos à vista é positivamente relacionada ao volume de recursos emprestados. A razão é que, independentemente do destino dos recursos, estes são computados como depósitos à vista para os agentes receptores do crédito (CARVALHO, 200o). Além disso, regiões que apresentam maiores volumes de crédito também apresentam maiores volumes de depósitos à vista. Tal evidência diz respeito, dentro da realidade nacional, às regiões mais desenvolvidas, como é o Sudeste brasileiro.

Quando testada a relação entre o dinamismo de cada economia estadual, medido pelo PIB estadual, e o volume de crédito, confirma-se a premissa de relação positiva, Isso sugere que, considerando-se a realidade do Sudeste, os valores crescentes do PIB estão associados às variações positivas no volume de crédito estadual. Este resultado é semelhante ao de Cavalcante, Crocco e Jayme Jr. (2006) em estudo a respeito do impacto da preferência pela liquidez sobre a oferta de crédito para os estados brasileiros entre 1988 a 1999.

Considerando-se a forma duplo-log, os estimadores representam elasticidades. Assim, o coeficiente positivo obtido na relação entre crédito e PIB estadual aponta que, diante da variação de $1 \%$ no PIB, em média, tem-se o aumento de 0,96\% no volume de crédito. Trata-se de um valor elevado e coerente com os estados analisados, considerando-se que as estatísticas apresentadas revelam que, de maneira geral, estes demonstram menores índices de preferência pela liquidez. Tal cenário proporciona aos agentes maior disponibilidade de recursos para financiamentos e empréstimos regionais. No que concerne à relação inversa entre PLB e crédito, é um resultado coerente com o argumento teórico de que o aumento da preferência bancária pode provocar redução da disponibilidade de crédito regional. O coeficiente obtido aponta que a elevação de $1 \%$ no índice proxy da PLB promove a diminuição em média de 0,72\% no volume de crédito. Por fim, enfatizando a influência dos depósitos totais sobre o crédito, pode-se inferir que a variação de $1 \%$ nos depósitos está relacionada às alterações positivas de aproximadamente $0,12 \%$ no crédito.

A seguir, na Tabela 4, o primeiro destaque diz respeito às relações positivas estatisticamente significativas entre as variáveis explicativas (patentes, PIB e crédito) e a variável dependente proxy do progresso tecnológico estadual (TEC). Observa-se que a variação de $1 \%$ no PIB tem impacto médio sobre a proxy de inovação em torno de $0,67 \%$. 
TORRES, D. A. R; VIEIRA, F. C.,; CRUZ, A. C. Sistema Financeiro, sistema de inovação e desenvolvimento....

TABELA 4 - MODELO DE EFEITOS FIXOS PARA EXPLICAR VARIAÇÕES NO NÍVEL DE PROGRESSO TECNOLÓGICO, ESTADOS DA REGIÃO SUDESTE, 2000-2012

\begin{tabular}{|c|c|}
\hline Variáveis Explicativas & Crédito \\
\hline Constante & $\begin{array}{c}-\mathbf{2 1}, 71^{* * *} \\
(2,0261)\end{array}$ \\
\hline Crédito & $\begin{array}{c}\mathbf{o , 3 8 6 4} 4^{* * *} \\
(0,2609)\end{array}$ \\
\hline Produto Interno Bruto & 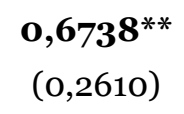 \\
\hline Patente $_{t-1}$ & $\begin{array}{c}\mathbf{o , 5 5 3 4} 4^{* * *} \\
(0,1537)\end{array}$ \\
\hline Coeficiente de determinação intraestados $\left(\mathrm{R}^{2}{ }_{\text {within }}{ }^{\prime}\right.$ & 0,8981 \\
\hline Coeficiente de determinação entre estados $\left(\mathrm{R}^{2}{ }_{\text {between }}\right)$ & 0,8632 \\
\hline Coeficiente de determinação geral $\left(\mathrm{R}^{2}{ }_{\text {overall }}\right)$ & 0,7803 \\
\hline Erro padrão de $\alpha_{i}$ & 1,5767 \\
\hline Erro padrão de $\varepsilon_{\text {is }}$ & 0,1066 \\
\hline Correlação entre os resíduos - $\operatorname{Corr}\left(\varepsilon_{\dot{\text { is }}}, \varepsilon_{i}\right)$ & 0,9954 \\
\hline Teste F de Significância Global & 120,44 \\
\hline
\end{tabular}

Fonte: Resultados da pesquisa.

Nota: Forma Funcional duplo - log. Valores entre parênteses: erro-padrão.

- ns: não significativo, ${ }^{* * *}$ : significativo a $1 \%,{ }^{* *}$ : significativo a $5 \% \mathrm{e}^{*}$ : significativo a $10 \%$.

Ademais, o crédito também tem impacto positivo sobre a criação de tecnologia e/ou inovação. Considerando-se que a preferência pela liquidez tem relação inversamente proporcional com o crédito destes estados, variável que por sua vez possui associação linear positiva com o progresso tecnológico dessas economias, pode-se inferir, mesmo que indireta, a relação entre preferência pela liquidez e progresso tecnológico das economias do Sudeste. Este resultado corrobora a base teórica deste estudo de que a menor a preferência pela liquidez dos bancos associa-se ao maior montante de crédito disponibilizado e ao desenvolvimento inovativo proporcionado intraestado.

Por fim, o modelo estimado indica que o número de patentes do período anterior também tem relação positiva com a proxy de patamar tecnológico de cada estado. Tal resultado demonstra que o learning-by-doing (patentes 
do período anterior) tem impacto positivo no volume de inovações, o que é corroborado em Romero e Jayme Jr. (2008).

Em suma, observa-se que os resultados empíricos obtidos confirmam a importância do crédito para o processo de inovação, conforme discutido por Schumpeter (1982). As estimativas auferidas atestam ainda a dependência dos sistemas de inovação de fontes de financiamento geradas pela expansão do crédito. Isso implica na necessidade de empresários inovadores schumpeterianos conseguirem convencer os bancos a fornecer o crédito para custear a inovação. Por um lado, vale lembrar que os bancos têm preferência pela liquidez, cuja escala varia segundo a confiança nas expectativas de retorno esperado dos diversos ativos. Por outro, Torres (2009) ressalta que, mesmo em cenários de expansão monetária internacional, os bancos nacionais priorizam projetos de investimento do tipo especulativo (ponzi) devido à vulnerabilidade externa estrutural junto a políticas monetárias ineficazes. Portanto, deve-se destacar que a expansão econômica, via crédito, não garante inovação de qualidade, ou mesmo início do processo de inovação.

Assim, a relação negativa entre PLB e crédito não pode ser desconsiderada, pois sugere que quanto maior a PLB, menor a disposição dos bancos em financiar os projetos de investimento, especialmente de atividades inovativas, dado o grau de incerteza elevado a elas associado. Se, portanto, os bancos são mais relutantes em conceder empréstimos de longo prazo nas regiões com sistema de inovação pouco eficiente, ao negar apoio suficiente à inovação, podem impedir ou limitar o fortalecimento do potencial dinâmico da economia.

Nestes termos, um caminho para o rompimento com o ciclo apresentado seria o amadurecimento dos seus sistemas de inovação. Este parece ser o caso da região Sudeste, haja vista a relação entre os níveis de atividade produtiva (medido pelo PIB) e de inovação. A relação das três variáveis citadas anteriormente parece demonstrar a construção e constituição de um ambiente propício à criação de tecnologia. Tal ambiente reduz a incerteza dos agentes quanto ao retorno de seus investimentos e concede a segurança esperada por agentes financeiros e bancos a investir no processo de inovação, considerado por Schumpeter (1982) força propulsora do crescimento econômico.

\section{Considerações Finais}

No que concerne às conclusões, é possível reconhecer fortes evidências de associação entre sistema financeiro dinâmico e um eficiente sistema de inovação estadual. As discussões teórica e empírica confirmam o sistema financeiro 
como parte integrante do sistema estadual de inovações. Ou seja, possuir um grau desenvolvido de conhecimento, produção científica e tecnológica integrado a um sistema financeiro maduro e eficiente traz maior dinamismo à economia nacional. Ademais, o estudo sugere o sistema financeiro como parte integrante do sistema nacional de inovações, de modo que um modesto desenvolvimento relativo do SI associa-se também a deficiências do sistema financeiro doméstico.

Todavia, não se pode inferir na análise da região Sudeste pela causalidade (uni ou bidirecional) entre sistema financeiro e sistema de inovação. O que observa é que, de maneira geral, tanto o sistema financeiro, quanto o sistema de inovação são altamente dependentes dos países centrais via indústrias transnacionais, montadoras e bancos privados internacionais. ${ }^{8}$ Neste contexto, destaca-se aqui a possibilidade de aprofundamento dos estudos a respeito da causalidade entre variáveis tecnológicas e financeiras, sobretudo, das regiões brasileiras, portanto, caracteriza como de linha de pesquisa futura.

Ressalta-se também a discrepância entre o estado de São Paulo e os demais estados do Sudeste, principalmente se comparado ao do Espírito Santo. Especificamente, o estado paulista destaca-se na oferta de capital financeiro, concentração esta ligada às diferenças na estrutura industrial da região. Esse estado apresenta maiores montantes de todos os grupos de crédito, assim como para as variáveis financeiras relacionadas à dimensão da economia local, como tamanho do ativo e depósitos à vista.

Em suma, é relevante frisar que o presente estudo contribui teórica e empiricamente para o reconhecimento da importância de se investir e fomentar as atividades de P\&D, além da necessidade de se investir melhor na articulação entre os setores de produção e conhecimento, a fim de proporcionar a interação para apropriação produtiva dos mesmos. Com isso, um sistema econômico dinâmico pode contribuir para o aumento dos ganhos dos bancos e agências financiadoras, diminuindo o risco de perdas, as taxas de juros e também a preferência pela liquidez, motivando a disponibilidade de crédito de longo prazo. $O$ trabalho também tem como mérito a base de dados utilizada de construção complexa, dado o formato de disponibilização de dados de volume de créditos e de artigos publicados pelas instituições de pesquisa.

\section{Referências}

Albuquerque, E. M. National systems of innovation and Non-OECD countries: notes about a rudimentary and tentative "typology". Brazilian Journal of Political Economy, v.19, nº4(76), October- December. 1999.

8 Para mais detalhes sobre causalidade entre sistema financeiro e sistema de inovação, ver Alves, Toyoshima e Torres (2014). 
Alves, F. F; Toyoshima, S. H; Torres, D. R. Fluxos Desiguais do Sistema Financeiro e Inovativo: uma análise das assimetrias entre países ricos e pobres. Análise Econômica, v. 32, n. 62; 2014.

Alves, K. Melissa. B. Inovação das Firmas e Financiamento à Inovação no Brasil: uma análise do período recente. Dissertação (Mestrado em Economia), Universidade do Estado do Rio de Janeiro, Rio de Janeiro, 2011.

BACEN- Banco Central. Disponível em: http://www.bacen.gov.br/. Acesso em: abril de 2013.

Carvalho, L. D. Endogeneidade Monetária, Crescimento Econômico e Distribuição de Renda: uma integração teórica da macrodinâmica pós-keynesiana. 2005. Dissertação (Mestrado em Economia), Universidade Federal do Paraná, Curitiba, 2005.

Carvalho, F. J. C. Sauza, F. E. P. Sicsú, J. Paula, L. F. R., Stuart, R. Economia monetária e financeira. teoria e política.Rio de Janeiro: Campus, 2000. $454 p$.

Carvalho, F. J. C. Mr. Keynes and the post Keynesians: principles of macroeconomics for a monetary production economy. Aldershot: Edward Elgar, 1992. 236p.

Cavalcante, A.; Crocco, M.; Jayme Jr., F. Preferência pela liquidez, sistema bancário e disponibilidade de crédito regional. In: Crocco, M.; Jayme Jr., F. Moeda e território: uma interpretação da dinâmica regional brasileira. Belo Horizonte: Autêntica, 2006. p.295-315

Chesnais, F; Sauviat, C. O Financiamento da inovação no regime global de acumulação dominado pelo capital financeiro. In: Lastres, H. M. M.; Cassiolato, J. E.; Arroio, A. (Orgs.) Conhecimento, sistemas de inovação e desenvolvimento. Rio de Janeiro: UFRJ, 2005. cap.5, p.161-220.

Cintra, M. A. M. Uma visão crítica da teoria da repressão financeira. Campinas: Unicamp, 1999.

Corder, S; Salles Filho, S. Aspectos conceituais do financiamento à Inovação. Revista Brasileira de Inovação, v. 5, n. 1, jan./jun., p. 33-76, 2006.

Crocco, M.; Cavalcante, A.; Castro, C. The behavior of Liquidity Preference of banks and public and regional development: the case of Brazil, Journal of Post Keynesian Economics, vol.28, n.2, p.217-40, 2005.

Dosi, G.; Freeman, C.; Fabiani, S. The process of economic development: introducing some stylized facts and theories on technologies, firms and institutions. Industrial and Corporate Change, v. 3, n. 1, 1994.

Dow, S. C. Money and the economic process. Aldershot: Edward Elgar, 1993. Cap 11.

Dow, S. C. Post Keynesian monetary theory for an open economy. Journal of Post Keynesian Economics, Armonk, v. 9, n. 2, p. 237-257, Winter, 1986/87.

Ernst, H. Global production networks and the changing geography of innovation system: implications for developing countries. East-west Center Working Papers - Economics Series, n. 9, November, 2000.

Greene, W. Econometric Analysis. 5rd. New York: Macmillan, 1026 p. 2002.

Gujarati, D. N. Econometria básica. Editora Campus, 812 p. 2006.

Hausman, J. A. Specification tests in econometrics. Econometrica, Chicago, v. 46, 
TORRES, D. A. R; VIEIRA, F. C.,; CRUZ, A. C. Sistema Financeiro, sistema de inovação e desenvolvimento....

n. 5, p.1251-1271, Sep. 1978.

Herskovic, B; Ribeiro, L. C; Albuquerque, E. M. Efeitos Recíprocos entre Finanças e Inovação. Belo Horizonte: UFMG/CEDEPLAR, 2008 (Texto para discussão $\left.n^{o} 332\right)$.

INPI - Instituto Nacional de Propriedade Industrial. Disponível em http://www. inpi.gov.br/portal/. Acesso em: abril 2013.

IPEADATA - Base de dados do Instituto de Política Econômica Aplicada. Disponível em: http://www.ipeadata.gov.br/. Acesso em: abril 2013.

ISI - Web Of Knowledge - IP \& Science. Disponível em: http://wokinfo.com/ . Acesso em: abril 2013.

Keynes, J. M. A teoria ex ante da taxa de juros. In: Fundação Instituto de Pesquisa Econômica Aplicada - IPEA. Clássicos da literatura econômica, Rio de Janeiro: IPEA/INPES, 1988b. 4o8p.

Keynes, J. M. A teoria geral do emprego, do juro e da moeda. São Paulo: Nova Cultural, 333p. 1983.

Keynes, J. M. Teorias alternativas da taxa de juros. In: Fundação Instituto de Pesquisa Econômica Aplicada - IPEA. Clássicos da literatura econômica, Rio de Janeiro: IPEA/INPES, 1988 a. 408p.

Keynes, J. M. (1937) A teoria geral do emprego. In: Szmrecsáiyi, T (Org.) Keynes, São Paulo: Àtica, 1999.

MICT - Ministério da Ciência e Tecnologia. Disponível em: http://www.mct.gov. br/. Acesso em: abril 2013.

Nelson, R. National innovation systems: a comparative analysis. New York, Oxford: Oxford University, 1993.

Nelson, R; Winter, S. An evolutionary theory of economic change. Cambridge: Harvard University, 1982.

Oliveira, G. B. Algumas considerações sobre Inovação Tecnológica, Crescimento Econômico e Sistemas Nacionais de Inovação. Rev. FAE, Curitiba, v.4, n.3, p.5-12, 2001.

Paula, L. F de. Financiamento, crescimento econômico e funcionalidade do sistema financeiro: uma abordagem pós-keynesiana. Estudos Econômicos (São Paulo), v. 43, n. 2, p. 363-396, 2013.

Paula, L. F. R. Teoria horizontalista da moeda e do crédito: crítica da crítica. Estudos Econômicos (IPE/USP), São Paulo, v.33, n.2, p.325-352, abr./jun. 2003.

Paula, L. F. Sistema Financeiro e o Financiamento da Inovação: Uma abordagem keynesiana-schumpeteriana. Encontro Nacional de Economia, v. 39, 2011.

Resende, M. F. C. O Padrão dos ciclos de crescimento da economia brasileira: 19472003. Economia e Sociedade, Campinas, v.14, n.1, p.109-129, jan./jun. 2005.

Romero, J. P; Jayme Jr., F. G. Sistema financeiro, inovação e desenvolvimento regional: Um estudo sobre a relação entre preferência pela liquidez e inovação no Brasil. Anais do XIV Encontro Regional de Economia do Nordeste, Fortaleza, 2008.

Silva, M. S. Sistema nacional de inovação e desempenho do setor externo. 2011. 
TORRES, D. A. R; VIEIRA, F. C.,; CRUZ, A. C. Sistema Financeiro, sistema de inovação e desenvolvimento....

Dissertação de Mestrado. Universidade Federal de Viçosa, Viçosa, 2011.

Sbicca, A; Pelaez, V. Sistemas de Inovação. In: Victor Pelaez e Tamás Szmerecsányi (org.). Economia da Inovação Tecnológica. São Paulo: Editora Hucitec, 2006.

Schumpeter, J. A. Teoria do desenvolvimento econômico. São Paulo: Abril Cultural, 1982. v.1. (Os Economistas).

Studart, R. Investment finance in economic development. London: Routledge, 1995. 234p.

Torres, D. A. R. Liquidez Internacional e Crescimento Econômico: Uma Análise Pós-Keynesiana Da Experiência Mundial. 2009. Tese (Doutorado em Economia), Universidade Federal de Minas Gerais (Cedeplar), Belo Horizonte, 2009.

Torres, D. A. R.; Resende, M, F. C. Causação circular do crescimento das economias em desenvolvimento: uma ponte entre as abordagens evolucionária e pós-keynesiana. Revista de Economia Política (Impresso), v. 33, p. 240-259, 2013.

Wooldrige, J. M. Introdução à Econometria: uma abordagem moderna. Tradução Ferreira, J. A. São Paulo, Cengage Learning, 2010. 
